\title{
Multi-cell cooperative transmission based on unitary space-time modulation
}

\author{
Heng Liu* ${ }^{*}$ Pingzhi Fan and Li Hao
}

\begin{abstract}
As a non-coherent transmission scheme that does not require channel state information at both transmitters and receivers, unitary space-time modulation is a promising technique that can be applied in high mobility scenario where the fading coefficients are changing too fast to be tracked and estimated. This article proposes a multi-cell cooperative transmission scheme based on unitary space-time modulation. Each cooperative base-station transmits an individual unitary signal from the common constellation set to the mobile unit which is located at the cell edge and suffers from severe inter-cell interference. Compared with traditional unitary space-time modulation, cooperation among multi-cells not only eliminates the inter-cell co-channel interference but also increases the transmission rate by expanding the constellation size. Performance of error probability is analyzed for the proposed scenario with maximum-likelihood decoding, in which the exact pairwise error probability is derived. Additionally, constellation optimization for cooperative transmission is also discussed to achieve the balance between transmission efficiency and reliability. Simulation results are provided to confirm the effectiveness of the proposed scheme in both block-fading channels and fast-fading channels.
\end{abstract}

\section{Introduction}

At present, the development of high-speed railway has put forward higher requirements for the wireless communication systems. The latest four-generation (4G) standard allows for a cellular mobile unit moving at speeds up to $350 \mathrm{~km} / \mathrm{h}$ [1]. Increasing commercial demand for broadband wireless communication to provide information and onboard entertainment services in the high-speed vehicles indicates that the network architecture, hardware devices and software algorithms should adapt to such super high-speed.

One major challenge of communications in high speed scenario is the time-selectivity caused by Doppler shifts or Doppler spread. Channel estimation becomes unrealistic in such time-variant channels, resulting the many of the current coherent reception techniques degrade in performance or even fail to work [2-4]. In 2000, Hochwald and Marzetta [5] investigate the capacity of Rayleigh flatfading channels where neither the transmitter nor the receiver knows the channel state information $(\mathrm{CSI})$ and

\footnotetext{
*Correspondence: xyxzgp@163.com

Key Lab of Information Coding \& Transmission, Institute of Mobile

Communications, Southwest Jiaotong University, Chengdu, Sichuan, 610031

P. R. China
}

propose a space-time modulation scheme. The constellation of proposed scheme consists of a set of unitary matrices, hence, the name unitary space-time modulation. Following the similar philosophy, Hochwald and Sweldens [6] and Hughes [7] present differential unitary space-time modulation, which is an extension of differential phaseshift keying (DPSK). All these studies are based on the assumption that channel-fading coefficients are constant over an entire block of $T$ temporal samples, however, a number of following studies shows that this non-coherent receiving scheme is still effective in the fast-fading channels, even when the mobile units are moving at very high speeds $[8,9]$.

This is the case of single link transmission. In the practical cellular network, all the cells share the same time and frequency channels, leading to the co-channel interference $(\mathrm{CCI})$ which greatly degrades the system performance. In the high-speed scenario, multi-cell CCI also exists and becomes more serve when the mobile units are moving across the cells or along the border area of the adjacent cells. Multi-base-stations (BSs) cooperation is believed to be the most effective way to eliminate the

\section{是 Springer}

(c) 2012 Liu et al: licensee Springer. This is an Open Access article distributed under the terms of the Creative Commons Attribution License (http://creativecommons.org/licenses/by/2.0), which permits unrestricted use, distribution, and reproduction in any medium, provided the original work is properly cited. 
inter-cell CCI [10-12]. However, most of current cooperative transmission schemes assume coherent transmission based on CSI exchange, which is impractical for the high-speed scenario. To avoid CSI exchange, this article proposes a multi-cell cooperative scenario by employing unitary space-time modulation, in which not only intercell CCI is eliminated in the fast-fading channel without CSI exchanging but also data rate is increased since all the cooperative BSs are transmitting individual data bits simultaneously. Error probability is analyzed in the proposed scenario and exact pairwise error probability (PEP) is derived for the general case, and the special case as well, in which the signals are mutually orthogonal. Based on the PEP results, cooperative constellation optimization is also considered to achieve the balance between the transmission rate and error performance. To the best of our knowledge, the multi-cell cooperative transmission based on unitary space-time modulation has not been treated before.

The rest of the article is organized as following: Section "Cooperative transmission based on unitary modulation" presents the system model, signal formulation and maximum-likelihood (ML) receiver for the cooperative transmission based on unitary space-time modulation. Performance analysis is in Section "Performance analysis" where exact PEP is derived for general case and special case. In Section "Cooperative constellation optimization", the cooperative constellation optimization is discussed. Simulation results are provided in Section "Simulation results". Section "Conclusion" concludes the article.

\section{Cooperative transmission based on unitary modulation \\ System model}

Consider a cellular network consisting of $K$ BSs, denoted as $B S_{1} \sim B S_{K}$ and each equipped with $M$ antennas. A mobile unit configured with $N$ antennas is moving at a very high speed in the cell-edge area. Suppose that signals are transmitted in blocks of $T$ successive symbols and each cooperative BS is transmitting an individual signal to the mobile unit. The channels are assumed to be Rayleigh flat-fading and constant within one block in the signal formulation, however in the simulation we also evaluate the realtime channels in which the fading coefficients are changing as a function of time. Channels from the $k$ th cooperative $\mathrm{BS}$ to the mobile unit are denoted by a $M \times N$ matrix $\mathbf{H}_{k}$ and all the elements within $\mathbf{H}_{k}$ are assumed to be independently complex Gaussian distributed with zeros mean and variance of $\sigma_{k}^{2}$. Obviously, $\sigma_{k}^{2}$ corresponds to the average power level of the link from the $k$ th BS to mobile unit influenced by the large-scale fading. A typical example of the system is depicted in Figure 1, in which two cooperative BSs are involved. The benefits of the cooperation are straightforward to be seen from Figure 1: the link from $B S_{2}$ which used to be interference link in the non-cooperative mode turns to be the desired link, not only eliminating the inter-cell CCI but also increasing the transmission rate.

\section{Signal formulation}

According to the system model mentioned above, for any block time, the received signals in baseband form can be represented by

$$
\mathbf{X}=\sqrt{\rho} \sum_{k=1}^{K} \mathbf{S}_{k} \mathbf{H}_{k}+\mathbf{W},
$$

where $\mathbf{S}_{k}(T \times M)$ is the signal from the $k$ th cooperative BS, $\mathbf{W}(T \times N)$ is the additive noise observed at the receiver and follows independently complex Gaussian distributed with zeros mean and unit variance, and $\rho$ represents the signal-to-noise ratio (SNR).

Conditioned on transmitted symbol $\mathbf{S}_{k}, k=1,2, \ldots, K$, the received signal $\mathbf{X}(T \times N)$ has independent and identically distributed columns (across the $N$ receiving antennas) [5]. At a particular antenna, the $T$ received symbols are zero-mean symmetric complex Gaussian, with $T \times T$ covariance matrix

$$
\boldsymbol{\Lambda}=\mathbf{I}_{T}+\frac{\rho}{M} \Sigma_{k=1}^{K} \sigma_{k}^{2} \mathbf{S}_{k} \mathbf{S}_{k}^{\dagger}
$$

where $\mathbf{I}_{T}$ is the $T \times T$ identity matrix and $\mathbf{A}^{\dagger}$ represents the conjugate transpose of $\mathbf{A}$.

Then the conditional probability density of the received signals is calculated as

$$
p\left(\mathbf{X} \mid\left\{\mathbf{S}_{1}, \mathbf{S}_{2}, \ldots, \mathbf{S}_{K}\right\}\right)=\frac{\exp \left(-\operatorname{tr}\left\{\mathbf{\Lambda}^{-1} \mathbf{X} \mathbf{X}^{\dagger}\right\}\right)}{\operatorname{det}^{N} \boldsymbol{\Lambda}},
$$

where $\operatorname{tr}\{\cdot\}$ denotes the trace function. Equation (3) provides a basis to design the ML receiver.

\section{$M L$ receiver}

We now consider the ML non-coherent reception of multi-cell unitary space-time modulated signals. Suppose the signals transmitted by different cooperative BSs are from the same constellation set $\mathcal{S}$, named root constellation set, which consists of $L$ signals, i.e.

$$
\mathbf{S}_{k} \in \mathcal{S}=\left\{\sqrt{T} \boldsymbol{\Phi}_{1}, \sqrt{T} \boldsymbol{\Phi}_{2}, \ldots, \sqrt{T} \boldsymbol{\Phi}_{L}\right\}
$$

in which $\boldsymbol{\Phi}_{l}, \forall l$, are $T \times M$ unitary matrices satisfying $\boldsymbol{\Phi}_{l}^{\dagger} \boldsymbol{\Phi}_{l}=\mathbf{I}$ and the scaling factor ensures that the transmitted signals meet the energy constraint so that $\frac{1}{M} E\left[\Sigma_{m=1}^{M}\left|\mathbf{S}_{k}\right|^{2}\right]=1$. By cooperative transmission, each cooperative BS sends an individual signal which is residing in the root constellation, thus the $K$ transmitted signals are actually the permutation and combination of the $L$ signals from the root constellation, which forms the cooperative constellation with the size of $L^{K}$, denoted as $\mathcal{C}$. Assume the multi-cell transmission signal is denoted by 


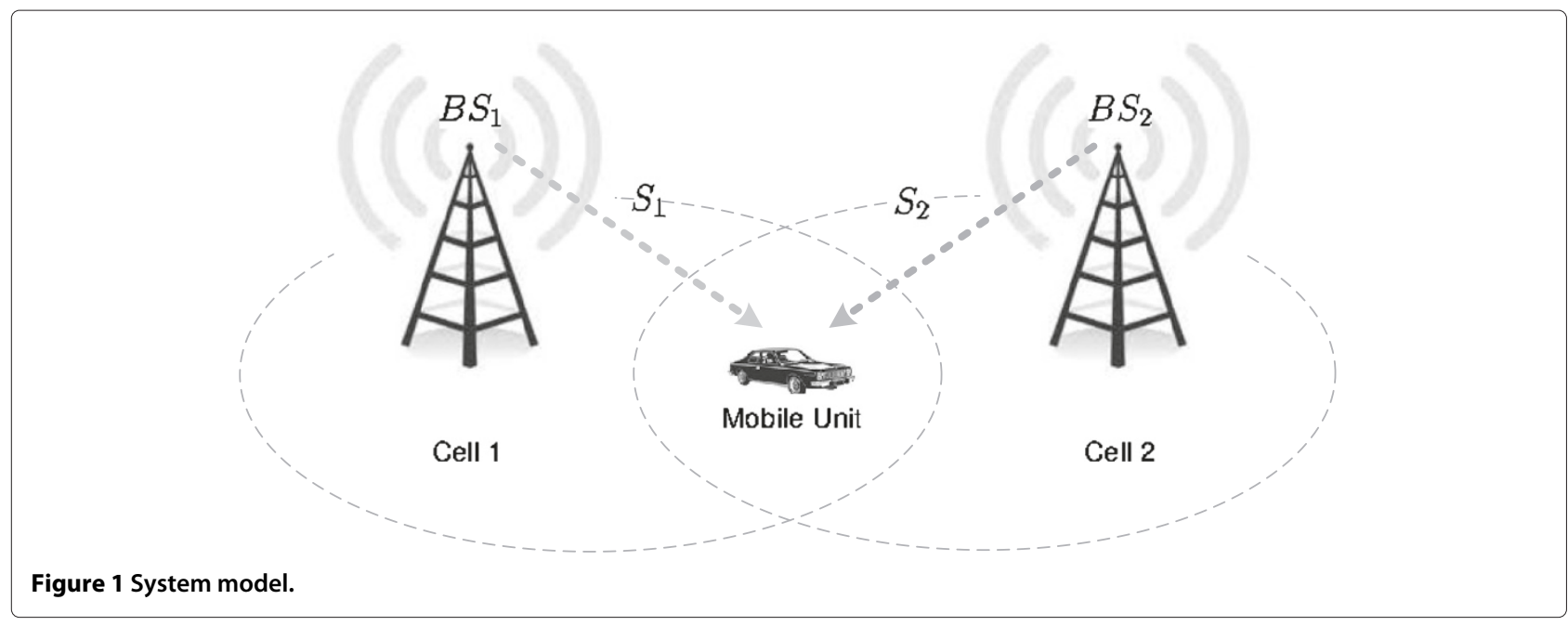

$\Omega=\left\{\mathbf{S}_{1}, \mathbf{S}_{2}, \ldots, \mathbf{S}_{K}\right\} \in \mathcal{C}$, then to perform the $\mathrm{ML}$ receiver, (3) is to be maximized and ML decoding becomes

$$
\begin{aligned}
\Omega_{o p t} & =\arg \max _{\mathbf{S}_{k} \in \mathcal{S}, \forall k} p\left(\mathbf{X} \mid\left\{\mathbf{S}_{1}, \mathbf{S}_{2}, \ldots, \mathbf{S}_{K}\right\}\right) \\
& =\arg \max _{\mathbf{S}_{k} \in \mathcal{S}, \forall k} \frac{\exp \left(-\operatorname{tr}\left\{\left[\mathbf{I}_{T}+\frac{\rho}{M} \Sigma_{k=1}^{K} \sigma_{k}^{2} \mathbf{S}_{k} \mathbf{S}_{k}^{\dagger}\right]^{-1} \mathbf{X} \mathbf{X}^{\dagger}\right\}\right)}{\pi^{T N} \operatorname{det}^{N}\left[\mathbf{I}_{T}+\frac{\rho}{M} \Sigma_{k=1}^{K} \sigma_{k}^{2} \mathbf{S}_{k} \mathbf{S}_{k}^{\dagger}\right]},
\end{aligned}
$$

which is optimal in the sense of ML receiver.

\section{Performance analysis}

The probability of decoding error for the multi-cell cooperative signals is analyzed in this section. The multi-cell signals within the cooperative constellation are no longer unitary, resulting in the more complicated PEP analysis compared with traditional unitary space-time modulation. The general case is firstly discussed, followed by the special case in which the unitary signals within the constellation set are mutually orthogonal. Pair-wise errors are categorized into three error patterns for the special case, which provides a baseline for the constellation optimization discussed in Section "Cooperative constellation optimization".

\section{General case}

Consider the case that the transmit signals from $K$ cooperative BSs are $\left\{\mathbf{S}_{1}, \ldots, \mathbf{S}_{K}\right\}$ while the detected signals are $\left\{\tilde{\mathbf{S}}_{1}, \ldots, \tilde{\mathbf{S}}_{K}\right\}$. The error probability is

$$
\begin{aligned}
& P_{\left\{\tilde{\mathbf{S}}_{1}, \ldots, \tilde{\mathbf{S}}_{K}\right\} \mid\left\{\mathbf{S}_{1}, \ldots, \mathbf{S}_{K}\right\}} \\
& =P\left\{\frac{e^{-\operatorname{tr}\left[\left(\mathbf{I}_{T}+\rho / M \sum_{k=1}^{K} \sigma_{k}^{2} \tilde{\mathbf{S}}_{k} \tilde{\mathbf{S}}_{k}^{\dagger}\right)^{-1} \mathbf{X} \mathbf{X}^{\dagger}\right]}}{\tilde{\Delta}}>\frac{e^{-\operatorname{tr}\left[\left(\mathbf{I}_{T}+\rho / M \sum_{k=1}^{K} \sigma_{k}^{2} \mathbf{S}_{k} \mathbf{S}_{k}^{\dagger}\right)^{-1} \mathbf{X} \mathbf{X}^{\dagger}\right]}}{\Delta}\right\},
\end{aligned}
$$

where $\tilde{\Delta}=\operatorname{det}^{N}\left(\mathbf{I}_{T}+\rho / M \sum_{k=1}^{K} \sigma_{k}^{2} \tilde{\mathbf{S}}_{k} \tilde{\mathbf{S}}_{k}^{\dagger}\right)$ and $\Delta=$ $\operatorname{det}^{N}\left(\mathbf{I}_{T}+\rho / M \sum_{k=1}^{K} \sigma_{k}^{2} \mathbf{S}_{k} \mathbf{S}_{k}^{\dagger}\right)$. Due to the property of unitary matrices, $\sum_{k=1}^{K} \sigma_{k}^{2} \mathbf{S}_{k} \mathbf{S}_{k}^{\dagger}$ and $\sum_{k=1}^{K} \sigma_{k}^{2} \tilde{\mathbf{S}}_{k} \tilde{\mathbf{S}}_{k}^{\dagger}$ can be decomposed as following

$$
\begin{aligned}
& \sum_{k=1}^{K} \sigma_{k}^{2} \mathbf{S}_{k} \mathbf{S}_{k}^{\dagger}=\mathbf{U} \boldsymbol{\Sigma} \mathbf{U}^{\dagger}, \\
& \sum_{k=1}^{K} \sigma_{k}^{2} \tilde{\mathbf{S}}_{k} \tilde{\mathbf{S}}_{k}^{\dagger}=\tilde{\mathbf{U}} \tilde{\mathbf{\Sigma}} \tilde{\mathbf{U}}^{\dagger},
\end{aligned}
$$

where both $\mathbf{U}$ and $\tilde{\mathbf{U}}$ are unitary matrices, both $\boldsymbol{\Sigma}$ and $\tilde{\boldsymbol{\Sigma}}$ are diagonal matrices. By taking (7) into (6) and invoking the matrix inversion lemma, (6) is written as

$$
P_{\left\{\tilde{\mathbf{S}}_{1}, \ldots, \tilde{\mathbf{S}}_{K}\right\} \mid\left\{\mathbf{S}_{1}, \ldots, \mathbf{S}_{K}\right\}}=P\left\{\kappa>\frac{M}{\rho} \log \frac{\tilde{\Delta}}{\Delta}\right\},
$$

where $\kappa$ is defined as $\operatorname{tr}\left\{\mathbf{X}^{\dagger}\left[\tilde{\mathbf{U}}\left(\tilde{\boldsymbol{\Sigma}}^{-1}+\rho / M \mathbf{I}_{T}\right)^{-1} \tilde{\mathbf{U}}^{\dagger}-\right.\right.$ $\left.\left.\mathbf{U}\left(\boldsymbol{\Sigma}^{-1}+\rho / M \mathbf{I}_{T}\right)^{-1} \mathbf{U}^{\dagger}\right] \mathbf{X}\right\}$. By using the vector notation, $\kappa$ can be further written as [13]

$$
\begin{aligned}
\kappa= & \operatorname{vec}(\mathbf{X})^{\dagger}\left[\left(\mathbf{I}_{T} \otimes \tilde{\mathbf{U}}\right)\left(\mathbf{I}_{T} \otimes \tilde{\mathbf{D}}\right)\left(\mathbf{I}_{T} \otimes \tilde{\mathbf{U}}\right)^{\dagger}\right. \\
& \left.-\left(\mathbf{I}_{T} \otimes \mathbf{U}\right)\left(\mathbf{I}_{T} \otimes \mathbf{D}\right)\left(\mathbf{I}_{T} \otimes \mathbf{U}\right)^{\dagger}\right] \operatorname{vec}(\mathbf{X}),
\end{aligned}
$$

where $\mathbf{D}=\left(\boldsymbol{\Sigma}^{-1}+\rho / M \mathbf{I}_{T}\right)^{-1}$ and $\tilde{\mathbf{D}}=\left(\tilde{\boldsymbol{\Sigma}}^{-1}+\rho / M \mathbf{I}_{T}\right)^{-1}$, $\otimes$ denotes the Kronecker product of two matrices, $\operatorname{vec}(\mathbf{X})$ is obtained by stacking the columns of $\mathbf{X}$ in the order from the first one to the last. According to (2) and (7), it is obvious that the covariance matrix of $\operatorname{vec}(\mathbf{X})$ is calculated as

$$
\mathbf{R}_{\mathbf{X}}=\mathbf{I}_{T N}+\rho / M\left(\mathbf{I}_{N} \otimes \mathbf{U}\right)\left(\mathbf{I}_{N} \otimes \mathbf{\Sigma}\right)\left(\mathbf{I}_{N} \otimes \mathbf{U}\right)^{\dagger} .
$$

Equation (8) can be determined by resorting to the characteristic function (CHF) of $\kappa$. Given that $\kappa$ is a quadratic 
form in Gaussian vector vec $(\mathbf{X})$, it is straightforward to obtain its $\mathrm{CHF}$ as

$$
E[\exp (s \kappa)]=\operatorname{det}\left(\mathbf{I}_{T N}-s \mathbf{D}_{\mathbf{X}} \mathbf{R}_{\mathbf{X}}\right)^{-1}=\frac{1}{\prod_{k=1}^{K}\left(1-r_{k} s\right)},
$$

where $r_{1}, r_{2}, \ldots, r_{K}$ are the eigenvalues of the matrix of $\mathbf{D}_{\mathbf{X}} \mathbf{R}_{\mathbf{X}}$ and $\mathbf{D}_{\mathbf{X}}=\left(\mathbf{I}_{T} \otimes \tilde{\mathbf{U}}\right)\left(\mathbf{I}_{T} \otimes \tilde{\mathbf{D}}\right)\left(\mathbf{I}_{T} \otimes \tilde{\mathbf{U}}\right)^{\dagger}-\left(\mathbf{I}_{T} \otimes \mathbf{U}\right)\left(\mathbf{I}_{T} \otimes\right.$ D) $\left(\mathbf{I}_{T} \otimes \mathbf{U}\right)^{\dagger}$. Consequently, the PEP for the general case is calculated as

$$
\begin{aligned}
P_{\left\{\tilde{\mathbf{S}}_{1}, \ldots, \tilde{\mathbf{S}}_{K}\right\} \mid\left\{\mathbf{S}_{1}, \ldots, \mathbf{S}_{K}\right\}} & =\frac{1}{2 \pi} \int_{0}^{\infty} \mathrm{d} s \int_{-\infty}^{+\infty} E[\exp (s \kappa)] \mathrm{d} \kappa \\
& =-\sum_{\operatorname{Re}\left(r_{k}\right)>0} \operatorname{Res}_{s=1 / r_{k}}\left\{\frac{e^{-\log \frac{\tilde{\Delta}}{\Delta} s}}{s \prod_{k=1}^{K}\left(1-r_{k} s\right)}\right\} .
\end{aligned}
$$

\section{Special case}

It is seen from (12) that the PEP expression for the general case is very complicated and not straightforward. Here, we extend the results to the special case, in which all the signals within the root constellation are mutually orthogonal to each other, i.e. $\boldsymbol{\Phi}_{i}^{\dagger} \boldsymbol{\Phi}_{j}=0, \forall i \neq j$. Two-cell cooperation with the root constellation size of 2 is considered as a typical example, in which the cooperative constellation generated from the root constellation is shown in Table 1. It can be easily extended to the scenarios with more cooperative cells or larger size of root constellation. To analyze the PEP, signal pairs are categorized into three patterns according to their different analysis methods, as shown in Table 2. The detailed PEP analysis will be derived based on different pairwise error patterns, respectively.

\section{Pairwise error Pattern I}

Pattern-I includes the pair of $\left\{\sqrt{T} \boldsymbol{\Phi}_{1}, \sqrt{T} \boldsymbol{\Phi}_{1}\right\}$ and $\left\{\sqrt{T} \boldsymbol{\Phi}_{2}, \sqrt{T} \boldsymbol{\Phi}_{2}\right\}$, indicating that both BSs transmit the same signals and detected signals are also the same. In this case, the $\mathbf{D}_{\mathbf{X}} \mathbf{R}_{\mathbf{X}}$ in (11) can be calculated as

$$
\begin{aligned}
\mathbf{D}_{\mathbf{X}} \mathbf{R}_{\mathbf{X}}= & \frac{\rho T\left(\sigma_{1}^{2}+\sigma_{2}^{2}\right) / M}{1+\rho T\left(\sigma_{1}^{2}+\sigma_{2}^{2}\right) / M}\left(\mathbf{I}_{N} \otimes \boldsymbol{\Phi}_{2}\right)\left(\mathbf{I}_{N} \otimes \boldsymbol{\Phi}_{2}\right)^{\dagger} \\
& -\rho T\left(\sigma_{1}^{2}+\sigma_{2}^{2}\right) / M\left(\mathbf{I}_{N} \otimes \boldsymbol{\Phi}_{1}\right)\left(\mathbf{I}_{N} \otimes \boldsymbol{\Phi}_{1}\right)^{\dagger} .
\end{aligned}
$$

Table 1 Two-cell cooperative constellation based on the root constellation size of 2

\begin{tabular}{lccl}
\hline Index & $\boldsymbol{B S}_{\mathbf{1}}$ transmits & $\boldsymbol{B} \boldsymbol{S}_{\mathbf{2}}$ transmits & $\begin{array}{l}\text { Cooperative } \\
\text { constellation } \mathcal{C}\end{array}$ \\
\hline 1 & $\sqrt{T} \boldsymbol{\Phi}_{1}$ & $\sqrt{T} \boldsymbol{\Phi}_{1}$ & $\left\{\sqrt{T} \boldsymbol{\Phi}_{1}, \sqrt{T} \boldsymbol{\Phi}_{1}\right\}$ \\
2 & $\sqrt{T} \boldsymbol{\Phi}_{2}$ & $\sqrt{T} \boldsymbol{\Phi}_{2}$ & $\left\{\sqrt{T} \boldsymbol{\Phi}_{2,} \sqrt{T} \boldsymbol{\Phi}_{2}\right\}$ \\
3 & $\sqrt{T} \boldsymbol{\Phi}_{1}$ & $\sqrt{T} \boldsymbol{\Phi}_{2}$ & $\left\{\sqrt{T} \boldsymbol{\Phi}_{1}, \sqrt{T} \boldsymbol{\Phi}_{2}\right\}$ \\
4 & $\sqrt{T} \boldsymbol{\Phi}_{2}$ & $\sqrt{T} \boldsymbol{\Phi}_{1}$ & $\left\{\sqrt{T} \boldsymbol{\Phi}_{2,} \sqrt{T} \boldsymbol{\Phi}_{1}\right\}$ \\
\hline
\end{tabular}

\section{Table 2 Pairwise error pattern categories}

\begin{tabular}{ll}
\hline Pairwise error pattern & \multicolumn{1}{c}{ Pairs include } \\
\hline II & $\left\{\sqrt{T} \boldsymbol{\Phi}_{1}, \sqrt{T} \boldsymbol{\Phi}_{1}\right\}\left\{\sqrt{T} \boldsymbol{\Phi}_{2}, \sqrt{T} \boldsymbol{\Phi}_{2}\right\}$ \\
& $\left\{\sqrt{T} \boldsymbol{\Phi}_{1}, \sqrt{T} \boldsymbol{\Phi}_{2}\right\}\left\{\sqrt{T} \boldsymbol{\Phi}_{2}, \sqrt{T} \boldsymbol{\Phi}_{1}\right\}$ \\
& $\left\{\sqrt{T} \boldsymbol{\Phi}_{1}, \sqrt{T} \boldsymbol{\Phi}_{1}\right\}\left\{\sqrt{T} \boldsymbol{\Phi}_{1}, \sqrt{T} \boldsymbol{\Phi}_{2}\right\}$, \\
& $\left\{\sqrt{T} \boldsymbol{\Phi}_{1}, \sqrt{T} \boldsymbol{\Phi}_{1}\right\}\left\{\sqrt{T} \boldsymbol{\Phi}_{2}, \sqrt{T} \boldsymbol{\Phi}_{1}\right\}$, \\
& III \\
& $\left\{\sqrt{T} \boldsymbol{\Phi}_{2}, \sqrt{T} \boldsymbol{\Phi}_{2}\right\}\left\{\sqrt{T} \boldsymbol{\Phi}_{1}, \sqrt{T} \boldsymbol{\Phi}_{2}\right\}$, \\
& $\left\{\sqrt{T} \boldsymbol{\Phi}_{2}, \sqrt{T} \boldsymbol{\Phi}_{2}\right\}\left\{\sqrt{T} \boldsymbol{\Phi}_{2}, \sqrt{T} \boldsymbol{\Phi}_{1}\right\}$.
\end{tabular}

Due to the orthogonal properties of the $\boldsymbol{\Phi}_{1}$ and $\boldsymbol{\Phi}_{2}$, the matrix $\mathbf{D}_{\mathbf{X}} \mathbf{R}_{\mathbf{X}}$ has $2 M N$ eigenvalues, $M N$ of which are $\frac{\rho T\left(\sigma_{1}^{2}+\sigma_{2}^{2}\right) / M}{1+\rho T\left(\sigma_{1}^{2}+\sigma_{2}^{2}\right) / M}$ and the rest $M N$ are $-\rho T\left(\sigma_{1}^{2}+\sigma_{2}^{2}\right) / M$. According to (12), the PEP of this error pattern is

$$
\begin{aligned}
& P_{\left\{\sqrt{T} \boldsymbol{\Phi}_{1}, \sqrt{T} \boldsymbol{\Phi}_{1}\right\} \mid\left\{\sqrt{T} \boldsymbol{\Phi}_{2}, \sqrt{T} \boldsymbol{\Phi}_{2}\right\}} \\
& =P_{\left\{\sqrt{T} \boldsymbol{\Phi}_{2}, \sqrt{T} \boldsymbol{\Phi}_{2}\right\} \mid\left\{\sqrt{T} \boldsymbol{\Phi}_{1}, \sqrt{T} \boldsymbol{\Phi}_{1}\right\}} \\
& =-\operatorname{Res}_{s=\frac{1+\rho T\left(\sigma_{1}^{2}+\sigma^{2}\right) / M}{\rho T\left(\sigma_{1}^{2}+\sigma_{2}^{2}\right) / M}} \\
& \times \frac{1}{s}\left\{\frac{1}{\left(1-\frac{\rho T\left(\sigma_{1}^{2}+\sigma_{2}^{2}\right) / M}{1+\rho T\left(\sigma_{1}^{2}+\sigma_{2}^{2}\right) / M} s\right)\left(1+\rho T\left(\sigma_{1}^{2}+\sigma_{2}^{2}\right) / M s\right)}\right\}^{M N} .
\end{aligned}
$$

In the case of $M=N=1,(14)$ can be further simplified as

$$
\begin{aligned}
P_{\left\{\sqrt{T} \boldsymbol{\Phi}_{1}, \sqrt{T} \boldsymbol{\Phi}_{1}\right\} \mid\left\{\sqrt{T} \boldsymbol{\Phi}_{2}, \sqrt{T} \boldsymbol{\Phi}_{2}\right\}} & =P_{\left\{\sqrt{T} \boldsymbol{\Phi}_{2}, \sqrt{T} \boldsymbol{\Phi}_{2}\right\} \mid\left\{\sqrt{T} \boldsymbol{\Phi}_{1}, \sqrt{T} \boldsymbol{\Phi}_{1}\right\}} \\
& =\frac{1}{2+\rho T\left(\sigma_{1}^{2}+\sigma_{2}^{2}\right)} .
\end{aligned}
$$

\section{Pairwise error Pattern II}

The pair falling into this pattern are the signals of $\left\{\sqrt{T} \boldsymbol{\Phi}_{1}, \sqrt{T} \boldsymbol{\Phi}_{2}\right\}$ and $\left\{\sqrt{T} \boldsymbol{\Phi}_{2}, \sqrt{T} \boldsymbol{\Phi}_{1}\right\}$. These two signals consist of the same root signals, but in different orders. The $\mathbf{D}_{\mathbf{X}} \mathbf{R}_{\mathbf{X}}$ in (11) can be calculated as

$$
\begin{aligned}
\mathbf{D}_{\mathbf{X}} \mathbf{R}_{\mathbf{X}}= & \frac{\rho T\left(\sigma_{1}^{2}-\sigma_{2}^{2}\right)}{M+\rho T \sigma_{2}^{2}}\left(\mathbf{I}_{N} \otimes \boldsymbol{\Phi}_{1}\right)\left(\mathbf{I}_{N} \otimes \boldsymbol{\Phi}_{1}\right)^{\dagger} \\
& +\frac{\rho T\left(\sigma_{2}^{2}-\sigma_{1}^{2}\right)}{M+\rho T \sigma_{2}^{2}}\left(\mathbf{I}_{N} \otimes \boldsymbol{\Phi}_{2}\right)\left(\mathbf{I}_{N} \otimes \boldsymbol{\Phi}_{2}\right)^{\dagger}
\end{aligned}
$$

which also has $2 M N$ eigenvalues, $M N$ of which are $\frac{\rho T\left(\sigma_{1}^{2}-\sigma_{2}^{2}\right)}{M+\rho T \sigma_{2}^{2}}$ and $M N$ are $\frac{\rho T\left(\sigma_{2}^{2}-\sigma_{1}^{2}\right)}{M+\rho T \sigma_{2}^{2}}$. Without loss of 
generality, assume $\sigma_{1}^{2} \geq \sigma_{2}^{2}$, therefore, the PEP of this error pattern is

$$
\begin{aligned}
& P_{\left\{\sqrt{T} \boldsymbol{\Phi}_{1}, \sqrt{T} \boldsymbol{\Phi}_{2}\right\} \mid\left\{\sqrt{T} \boldsymbol{\Phi}_{2}, \sqrt{T} \boldsymbol{\Phi}_{1}\right\}} \\
& =P_{\left\{\sqrt{T} \boldsymbol{\Phi}_{2}, \sqrt{T} \boldsymbol{\Phi}_{1}\right\} \mid\left\{\sqrt{T} \boldsymbol{\Phi}_{1}, \sqrt{T} \boldsymbol{\Phi}_{2}\right\}} \\
& =-\operatorname{Res}{ }_{s=\frac{M+\rho T \sigma_{2}^{2}}{\rho T\left(\sigma_{1}^{2}-\sigma_{2}^{2}\right)} \frac{1}{s}} \\
& \quad \times\left\{\frac{1}{\left(1-\frac{\rho T\left(\sigma_{1}^{2}-\sigma_{2}^{2}\right)}{M+\rho T \sigma_{2}^{2}} s\right)\left(1-\frac{\rho T\left(\sigma_{2}^{2}-\sigma_{1}^{2}\right)}{M+\rho T \sigma_{2}^{2}} s\right)}\right\}^{M N} .
\end{aligned}
$$

In the case of $M=N=1$, Equation (17) can be further simplified as

$$
\begin{aligned}
P_{\left\{\sqrt{T} \boldsymbol{\Phi}_{1}, \sqrt{T} \boldsymbol{\Phi}_{2}\right\} \mid\left\{\sqrt{T} \boldsymbol{\Phi}_{2}, \sqrt{T} \boldsymbol{\Phi}_{1}\right\}} & =P_{\left\{\sqrt{T} \boldsymbol{\Phi}_{2}, \sqrt{T} \boldsymbol{\Phi}_{1}\right\} \mid\left\{\sqrt{T} \boldsymbol{\Phi}_{1}, \sqrt{T} \boldsymbol{\Phi}_{2}\right\}} \\
& =\frac{1+\rho T \sigma_{1}^{2}}{2+\rho T\left(\sigma_{1}^{2}+\sigma_{2}^{2}\right)} .
\end{aligned}
$$

This is under the assumption of $\sigma_{2}^{2} \geq \sigma_{1}^{2}$. Similarly, in the case of $\sigma_{1}^{2} \geq \sigma_{2}^{2}$, the PEP becomes

$$
\begin{aligned}
P_{\left\{\sqrt{T} \boldsymbol{\Phi}_{1}, \sqrt{T} \boldsymbol{\Phi}_{2}\right\} \mid\left\{\sqrt{T} \boldsymbol{\Phi}_{2}, \sqrt{T} \boldsymbol{\Phi}_{1}\right\}} & =\frac{1+\rho T \sigma_{2}^{2}}{2+\rho T\left(\sigma_{1}^{2}+\sigma_{2}^{2}\right)} \\
& =P_{\left\{\sqrt{T} \boldsymbol{\Phi}_{2}, \sqrt{T} \boldsymbol{\Phi}_{1}\right\} \mid\left\{\sqrt{T} \boldsymbol{\Phi}_{1}, \sqrt{T} \boldsymbol{\Phi}_{2}\right\}}
\end{aligned}
$$

\section{Pairwise error Pattern III}

Consider the case that $\left\{\sqrt{T} \boldsymbol{\Phi}_{1}, \sqrt{T} \boldsymbol{\Phi}_{1}\right\}$ is transmitted when $\left\{\sqrt{T} \boldsymbol{\Phi}_{1}, \sqrt{T} \boldsymbol{\Phi}_{2}\right\}$ is detected. The $\mathbf{D}_{\mathbf{X}} \mathbf{R}_{\mathbf{X}}$ in (11) can be calculated as

$$
\begin{aligned}
\mathbf{D}_{\mathbf{X}} \mathbf{R}_{\mathbf{X}}= & -\frac{\rho T \sigma_{2}^{2}}{M+\rho T \sigma_{1}^{2}}\left(\mathbf{I}_{N} \otimes \boldsymbol{\Phi}_{1}\right)\left(\mathbf{I}_{N} \otimes \boldsymbol{\Phi}_{1}\right)^{\dagger} \\
& +\frac{\rho T \sigma_{2}^{2}}{M+\rho T \sigma_{2}^{2}}\left(\mathbf{I}_{N} \otimes \boldsymbol{\Phi}_{2}\right)\left(\mathbf{I}_{N} \otimes \boldsymbol{\Phi}_{2}\right)^{\dagger}
\end{aligned}
$$

whose eigenvalues consist of $M N$ of $\frac{\rho T \sigma_{2}^{2}}{M+\rho T \sigma_{2}^{2}}$ and $M N$ of $-\frac{\rho T \sigma_{2}^{2}}{M+\rho T \sigma_{1}^{2}}$. Consequently, the PEP of this error pattern is

$$
\begin{aligned}
& P_{\left\{\sqrt{T} \boldsymbol{\Phi}_{1}, \sqrt{T} \boldsymbol{\Phi}_{2}\right\} \mid\left\{\sqrt{T} \boldsymbol{\Phi}_{1}, \sqrt{T} \boldsymbol{\Phi}_{1}\right\}} \\
& =-\operatorname{Res}{ }_{s=\frac{M+\rho T \sigma_{2}^{2}}{\rho T \sigma_{2}^{2}} \frac{e^{-\log \frac{\tilde{\Delta}}{\Delta} s}}{s}} \\
& \quad \times\left\{\frac{1}{\left(1-\frac{\rho T \sigma_{2}^{2}}{M+\rho T \sigma_{2}^{2}} s\right)\left(1+\frac{\rho T \sigma_{2}^{2}}{M+\rho T \sigma_{1}^{2}} s\right)}\right\}^{M N} .
\end{aligned}
$$

For the special case that $M=N=1$, (21) can be simplified as

$$
P_{\left\{\sqrt{T} \boldsymbol{\Phi}_{1}, \sqrt{T} \boldsymbol{\Phi}_{2}\right\} \mid\left\{\sqrt{T} \boldsymbol{\Phi}_{1}, \sqrt{T} \boldsymbol{\Phi}_{1}\right\}}=\frac{\left(1+\rho T \sigma_{1}^{2}\right) e^{-\log \frac{\tilde{\Delta}}{\Delta} \frac{1+\rho T \sigma_{2}^{2}}{\rho T \sigma_{2}^{2}}}}{2+\rho T \sigma_{1}^{2}+\rho T \sigma_{2}^{2}} .
$$

Unlike the signal pairs of previous two patterns, the error probability when $\left\{\sqrt{T} \boldsymbol{\Phi}_{1}, \sqrt{T} \boldsymbol{\Phi}_{2}\right\}$ is transmitted but $\left\{\sqrt{T} \boldsymbol{\Phi}_{1}, \sqrt{T} \boldsymbol{\Phi}_{1}\right\}$ is received is not the same as (22) due to the asymmetrical structure of these two signals. However, following the same steps, the PEP for this case when $M=N=1$ is calculated as

$$
P_{\left\{\sqrt{\mathbf{T}} \boldsymbol{\Phi}_{1}, \sqrt{T} \boldsymbol{\Phi}_{1}\right\} \mid\left\{\sqrt{T} \boldsymbol{\Phi}_{1}, \sqrt{T} \boldsymbol{\Phi}_{2}\right\}}=1-\frac{\left(1+\rho T \sigma_{1}^{2}+\rho T \sigma_{2}^{2}\right) e^{-\log \frac{\tilde{\Delta}}{\Delta} \frac{1}{\rho T \sigma_{2}^{2}}}}{2+\rho T \sigma_{1}^{2}+\rho T \sigma_{2}^{2}} .
$$

\section{Cooperative constellation optimization}

For any types of modulation, the transmission rate $R$ is determined by the constellation size $Z$ in $R=\log _{2} Z$. Compared with the root constellation,the size of cooperative constellation increases from $Z=L$ to $Z=L^{K}$, leading to the increase of transmission rate. However, as it is well known, efficiency and reliability are two sides of the communication, which are hardly to obtain simultaneously. Increase in constellation size may result in the closer Euclidean distance among signals, which degrades the transmission reliability. In this section, we will discuss the cooperative constellation optimization problem to achieve the balance between the transmission rate and symbol-error rate (SER) performance. General speaking, some of the signals, which cause high error probability or even detection failure, will be removed from the signal constellation. For the ideal case, the goal of the optimization is to minimize the overall SER given any target transmission rate fulfilled. In other words, following optimization problem should be solved

$$
\begin{aligned}
& \mathcal{C}_{\text {opt }}=\arg \min _{\mathcal{C}_{i} \subseteq \mathcal{C}} P S \\
& \text { s.t. } \quad\left|\mathcal{C}_{i}\right|>\hat{Z},
\end{aligned}
$$

where $P s$ denotes the SER, $\hat{Z}$ is the target constellation size and $|\cdot|$ is the cardinality of a set. To solve this problem, one solution is the exhausting searching by testing all the subsets and looking for the optimal one. However, it can be computationally cumbersome when $|\mathcal{C}|$ goes large. Actually, based on the error probability analysis in Section "Performance analysis", some simple methods can be followed in the two-cell cooperation. Consider the special case and assume $M=N=1$, from the PEP equation in previous section, it is easy to identify that PEP is highest in the error Pattern-II, and the lowest in error Pattern-I, 


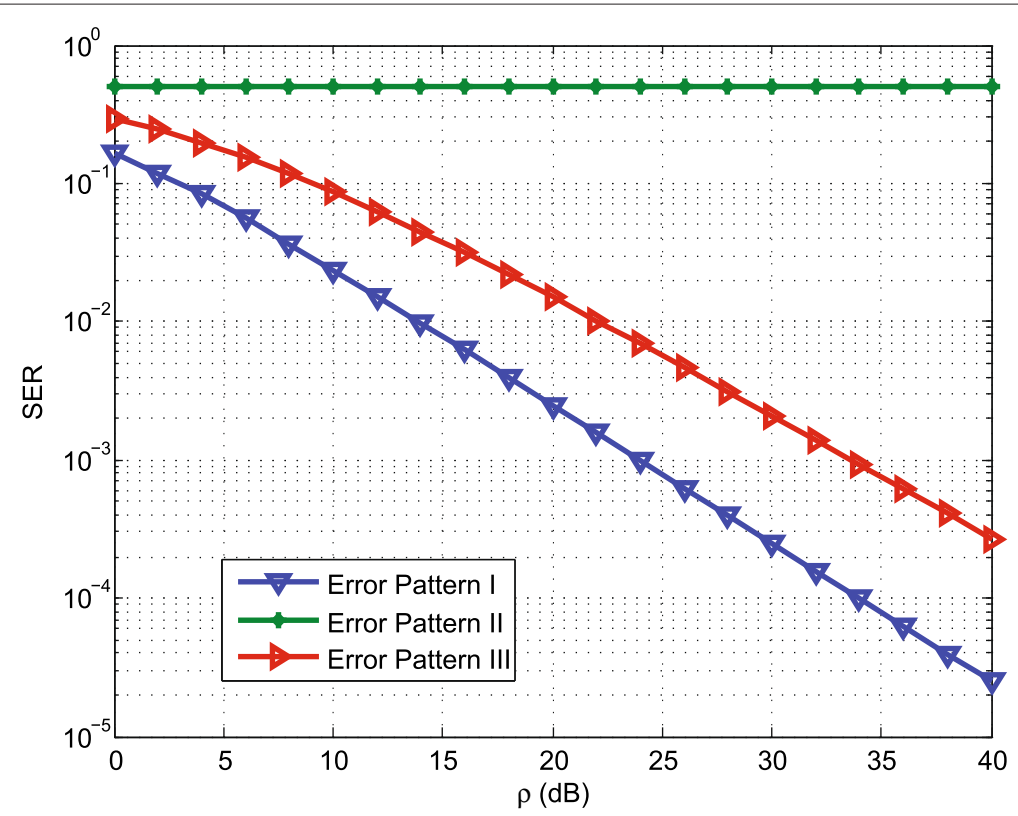

Figure 2 Numerical results of PEP.

which can be further confirmed in Figure 2. This indicates the order of signal discarding in the process of the constellation optimization: to avoid Pattern-II error takes first priority, then the error Pattern-III and finally error Pattern-I. Table 3 gives an example of signal optimization based on the cooperative constellation presented in Table 1, when the target constellation sizes $Z$ are set to 3 and 2, respectively. In this example, it is obvious to see when $Z=3$ the Pattern-II pairwise error is prevented and when $Z=2$ both Patterns-II and III pairwise errors are prevented.

After the optimization, the SER performance may be upper-bounded in terms of PEP through the union bound [14]

$$
\begin{aligned}
P_{s} & =\frac{1}{Z} \sum_{z=1}^{Z} P\left\{\operatorname{error} \mid\left\{\mathbf{S}_{1}, \ldots, \mathbf{S}_{K}\right\}\right\} \\
& =\frac{1}{Z} \sum_{z=1}^{Z} \sum_{\left\{\tilde{\mathbf{S}}_{1}, \ldots, \tilde{\mathbf{S}}_{K}\right\} \neq\left\{\mathbf{S}_{1}, \ldots, \mathbf{S}_{K}\right\}} P_{\left\{\tilde{\mathbf{S}}_{1}, \ldots, \tilde{\mathbf{S}}_{K}\right\} \mid\left\{\mathbf{S}_{1}, \ldots, \mathbf{S}_{K}\right\}} .
\end{aligned}
$$

Table 3 Two-cell cooperative constellation optimization based on the root constellation size of 2

\begin{tabular}{llllc}
\hline Index & $\begin{array}{l}\boldsymbol{B} \boldsymbol{S}_{\mathbf{1}} \\
\text { transmits }\end{array}$ & $\begin{array}{l}\boldsymbol{B} \boldsymbol{S}_{\mathbf{2}} \\
\text { transmits }\end{array}$ & $\mathcal{C}_{\text {opt }}, \boldsymbol{Z}=\mathbf{3}$ & $\mathcal{C}_{\text {opt }}, \boldsymbol{Z}=\mathbf{2}$ \\
\hline 1 & $\sqrt{T} \boldsymbol{\Phi}_{1}$ & $\sqrt{T} \boldsymbol{\Phi}_{1}$ & $\left\{\sqrt{T} \boldsymbol{\Phi}_{1}, \sqrt{T} \boldsymbol{\Phi}_{1}\right\}$ & $\left\{\sqrt{T} \boldsymbol{\Phi}_{1} \sqrt{T} \boldsymbol{\Phi}_{1}\right\}$ \\
2 & $\sqrt{T} \boldsymbol{\Phi}_{2}$ & $\sqrt{T} \boldsymbol{\Phi}_{2}$ & $\left\{\sqrt{T} \boldsymbol{\Phi}_{2}, \sqrt{T} \boldsymbol{\Phi}_{2}\right\}$ & $\left\{\sqrt{T} \boldsymbol{\Phi}_{2}, \sqrt{T} \boldsymbol{\Phi}_{2}\right\}$ \\
3 & $\sqrt{T} \boldsymbol{\Phi}_{1}$ & $\sqrt{T} \boldsymbol{\Phi}_{2}$ & $\left\{\sqrt{T} \boldsymbol{\Phi}_{1}, \sqrt{T} \boldsymbol{\Phi}_{2}\right\}$ & - \\
\hline
\end{tabular}

\section{Simulation results}

In the section, the performance of the cooperative transmission based on unitary space-time modulation will be evaluated by using Monte Carlo simulations. Different channel models are considered with respect to the speeds of the mobile unit, as well as the different symbol periods. For the root constellation construction, only the signals which are mutually orthogonal will be selected.

Block-constant channels are first considered, which provides the lower bounds in terms of SER performance. Assume two-cell cooperation in which $M=N=1$ and $T=2$. Figure 3 shows the results in the scenario where the link average power from two cooperative BSs to the mobile unit are asymmetrical, i.e. $\sigma_{1}^{2}=1, \sigma_{2}^{2}=0.32$, corresponding to 0 and $-5 \mathrm{~dB}$ of average power, respectively. In comparison, the traditional unitary space-time modulation without cooperation is also simulated. For this non-cooperative transmission, $B S_{1}$ works as the serving $\mathrm{BS}$ and $B S_{2}$ is the neighbor $\mathrm{BS}$ which causes inter-cell CCI to the mobile unit. It is seen from Figure 3 that without cooperation, $\mathrm{CCI}$ becomes the dominant factor restricting the SER performance instead of AWGN, leading to an error floor in the SER-versus-SNR curve. Meanwhile, the cooperative transmission without optimization also exhibits an error floor in high SNR region. This can be explained by the fact that for the error Pattern-II the PEP in (18) and (19) converges to certain constant instead of zero when SNR $\rho$ goes to infinite. Cooperative transmission with constellation optimization shows great advantage in SER performance, and $10 d B$ gain can be achieved by further reducing constellation size $Z$ from 3 


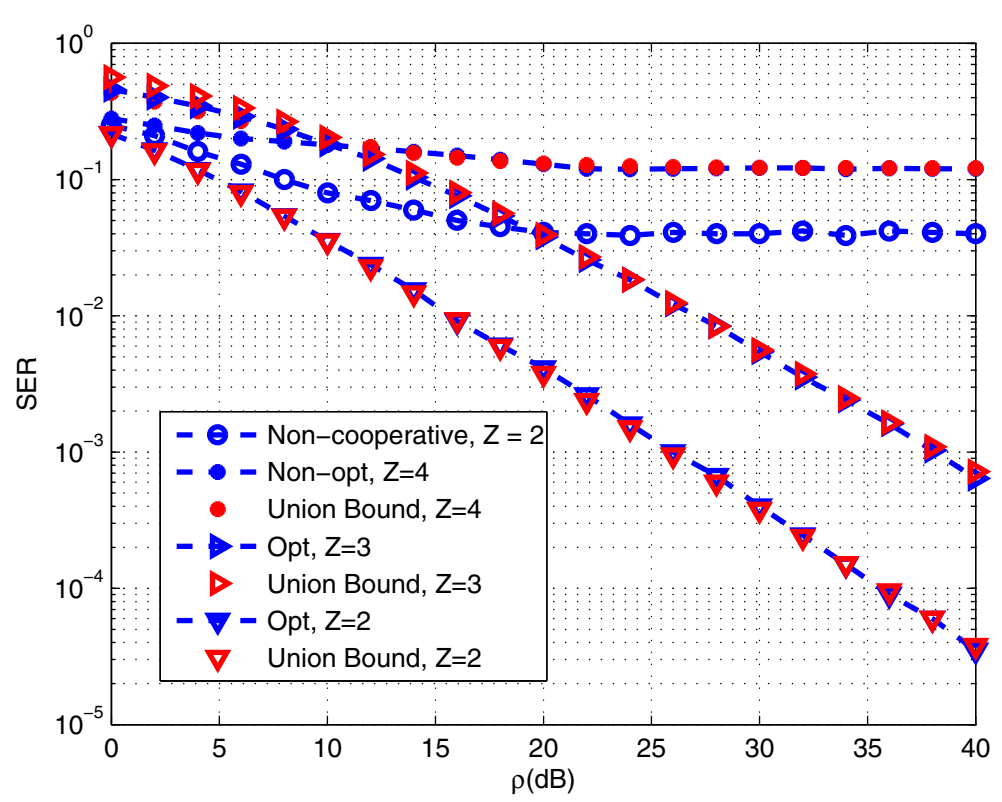

Figure 3 SER-versus-SNR. Block constant channels, $T=2, \sigma_{1}^{2}=1, \sigma_{2}^{2}=0.32$.

to 2. Besides the simulation results, the SER union bounds computed from (25) are also plotted in Figure 3, in which it is seen that the simulated SER are well-bounded by the SER union bounds. When the link power levels from the two cooperative BSs are equal, i.e. $\sigma_{1}^{2}=\sigma_{2}^{2}=1$, same simulation is performed as the results shown in Figure 4. Non-cooperative case degrades in performance and has even worse error floor due to the increase level of CCI. On the contrast, the SER performance with optimized cooperation improves by small degrees since both BSs transmits desired signals instead of interference. Figure 5 provides the SER results in terms of the constellation size $Z$, in which the symbol period $T$ increases to 5 and SNR $\rho$ is set to $25 \mathrm{~dB}$. As expected, in Figure 5 the SER performance degrades as the constellation size $Z$ increases. Two critical points happen when $Z=5$ and $Z=15$, in which the Pattern-III and Pattern-II errors are involved respectively, leading to the sharp increasing of SER.

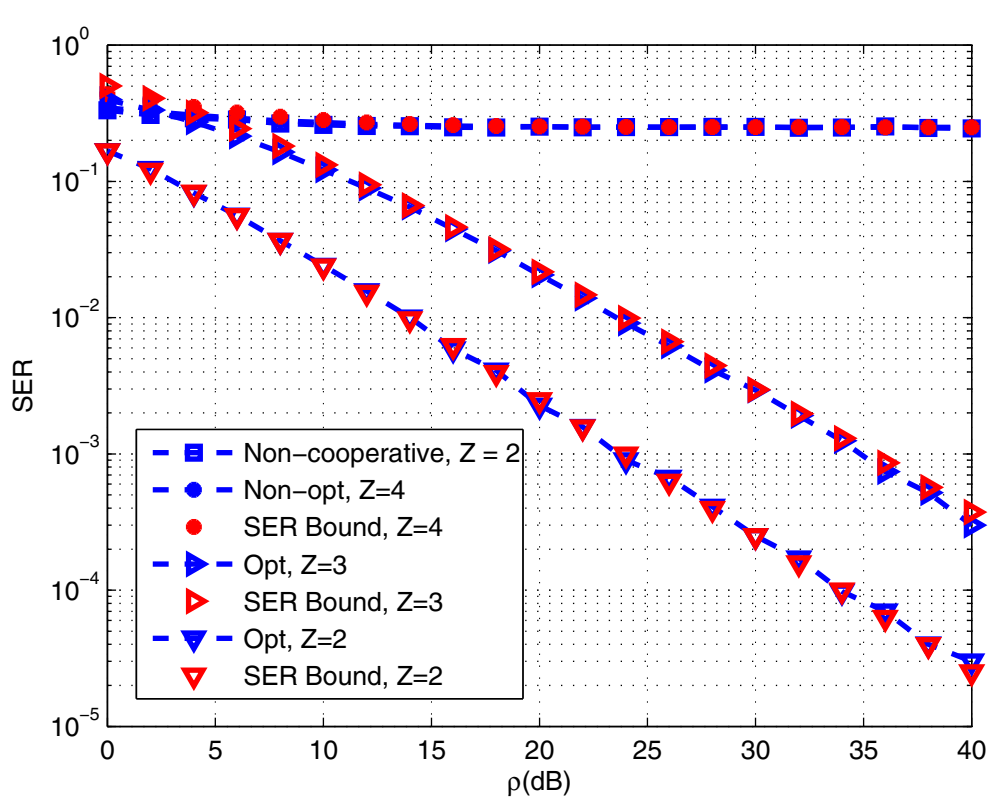

Figure 4 SER-versus-SNR. Block constant channels, $T=2, \sigma_{1}^{2}=\sigma_{2}^{2}=1$. 


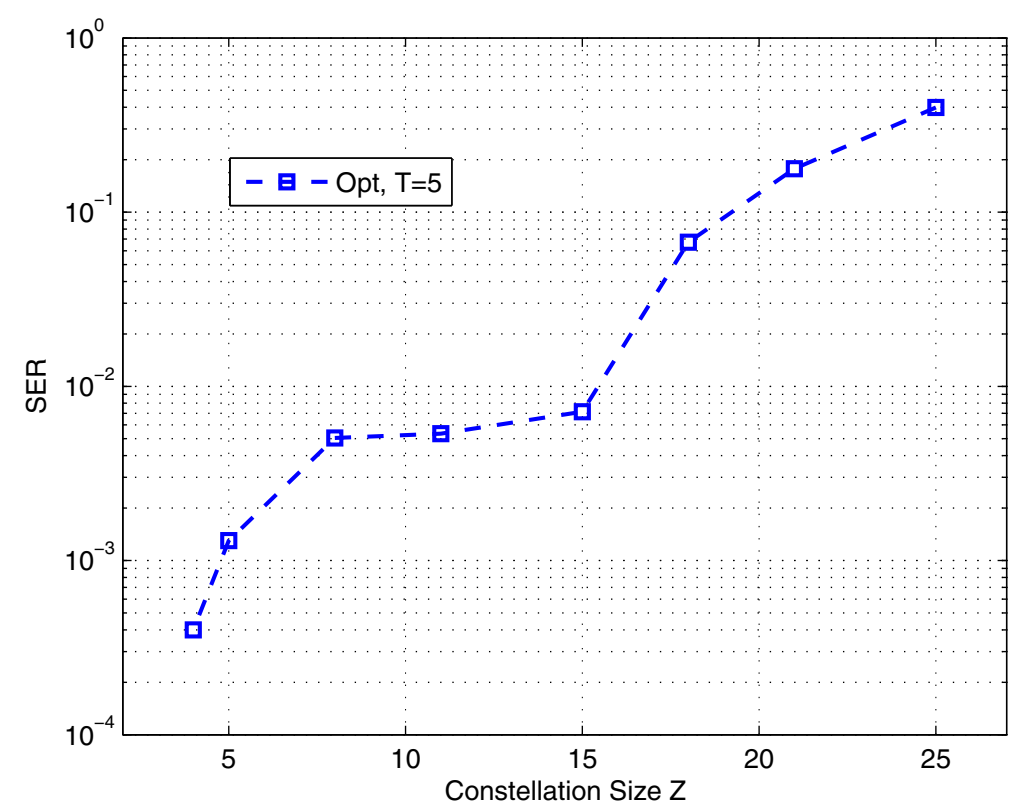

Figure 5 SER-versus-Z. Block constant channels, $T=5, \rho=25 \mathrm{~dB}$.

The fast fading channels are considered next. According to the Jakes model [15], the autocorrelation function of the fading coefficients in wireless channels can be represented by the 0th-order Bessel function of the first kind, i.e. $J_{0}\left(2 \pi f_{d} T_{s} t\right)$, where $T_{s}$ is the chip interval and $f_{d}$ corresponds to the maximum Doppler shift which can be further denoted by the carrier wavelength $\lambda$ and velocity $v$ as $f_{d}=\frac{v}{\lambda}$. During the simulation, the carrier frequency and chip rate are set to $2,400 \mathrm{MHz}$ and $100 \mathrm{kBd}$, respectively. SER-versus-SNR curves are plotted in Figure 6, where the velocity of the mobile unit is assumed to be $400 \mathrm{~km} / \mathrm{h}$ resulting in the correlation of 0.9992 between two chips within one symbol when $T=2$ and 0.9876 between the first and last chips when $T=5$, respectively. As comparison, the results under block-constant channels are also plotted. From Figure 6, it is seen the

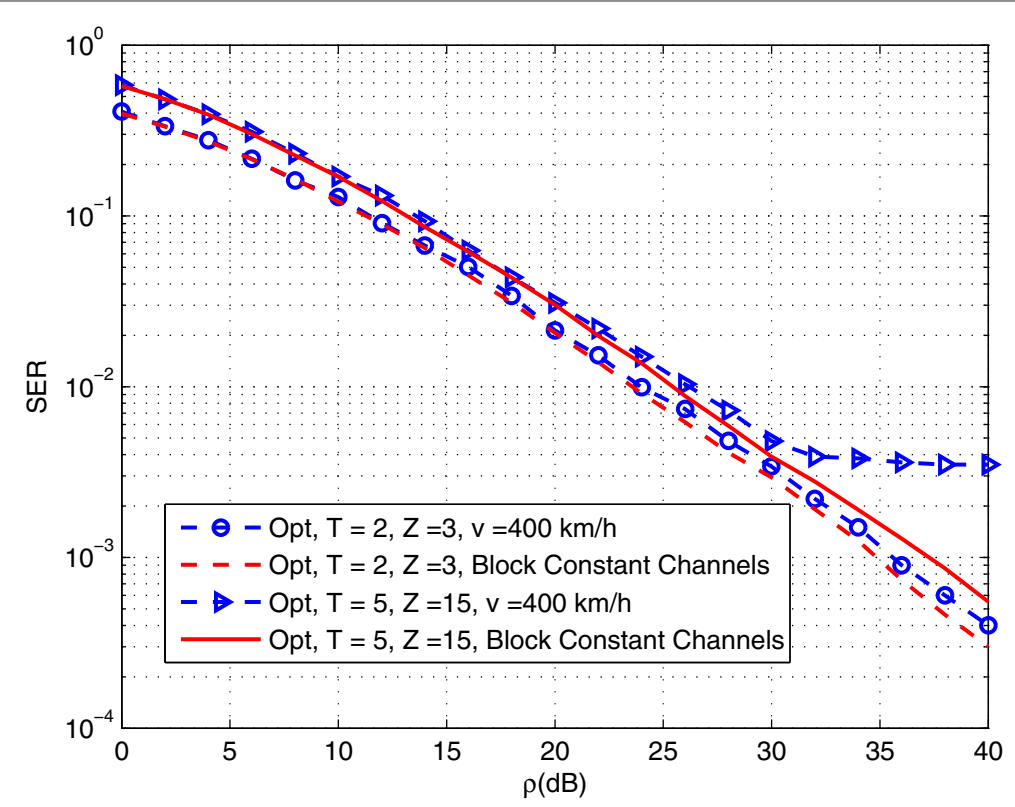

Figure 6 SER-versus-SNR. Time-variant channels, $v=400 \mathrm{~km} / \mathrm{h}, \sigma_{1}^{2}=\sigma_{2}^{2}=1$. 


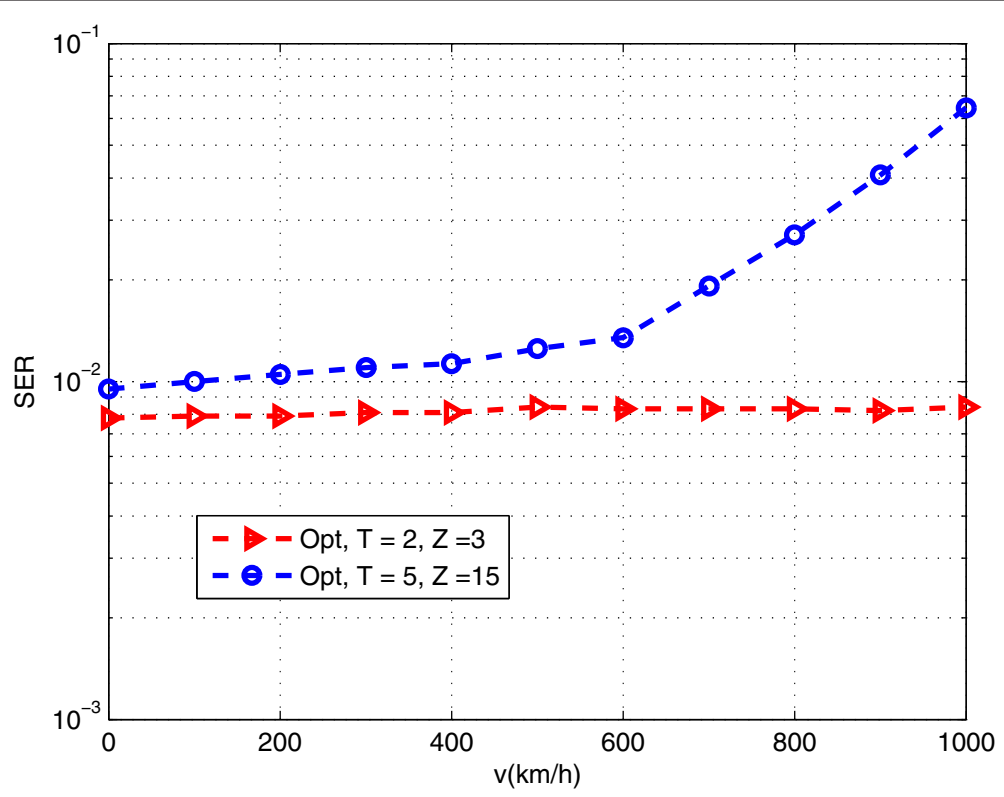

Figure 7 SER-versus-speed. $\rho=25 \mathrm{~dB}, \sigma_{1}^{2}=\sigma_{2}^{2}=1$.

SER performance almost shows no difference as in blockconstant channels when $T=2$, when $T=5$, the SER complies with the block-constant channels at the low SNR region, however, exhibits an error floor when $\rho$ increases to $30 \mathrm{~dB}$. Figure 7 plots the SER curves as a function of velocity in the case of $\rho=25 \mathrm{~dB}$. When $T=2$, the SER performance remains the same level even when the speed increases to $1,000 \mathrm{~km} / \mathrm{h}$, and in the case of $T=5$, SER also rises modestly until $v>600 \mathrm{~km} / \mathrm{h}$.
We also extend the proposed scheme to the doublyselective channels, in which the unitary space-time modulation can work as the unitary space-frequency modulation by utilizing orthogonal frequency division multiplexing (OFDM) [16]. In the simulation, the carrier frequency and velocity of the mobile unit are still assumed to be $2,400 \mathrm{MHz}$ and $400 \mathrm{~km} / \mathrm{h}$, respectively. OFDM with 2048 sub-carriers is exploited and the symbol duration $T_{s}$ is assumed to be $0.5 / 7 \mathrm{~ms}$, which complies with the frame

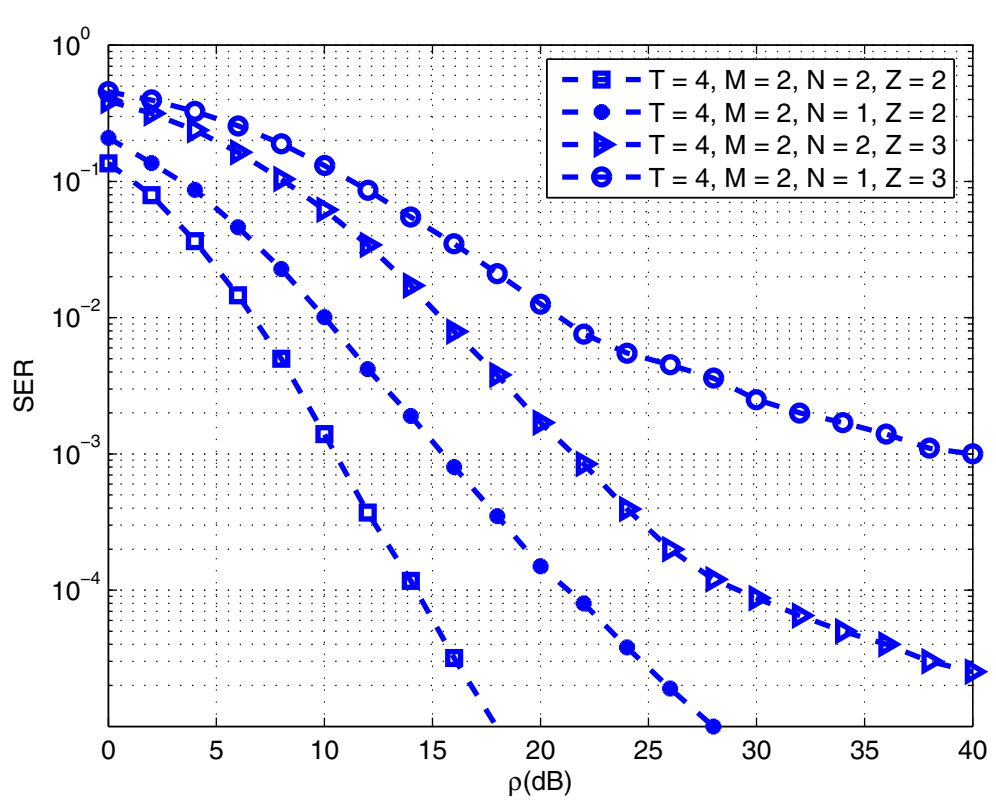

Figure 8 SER-versus-SNR. Doubly-selective channel, $\sigma_{1}^{2}=\sigma_{2}^{2}=1, v=400 \mathrm{~km} / \mathrm{h}$, OFDM with 2048 sub-carriers. 
structure of long term evolution (LTE) [17]. Figure 8 provides the SER-versus-SNR curves, in which it is seen that the proposed scheme is still effective under the doublyselective channels, though suffering from the error floor at the high SNR region which can be explained by the fact that time selectivity causes inter-carrier-interference (ICI) which restricts SER performance at the high-SNR region. Besides, more receiving antennas lead to more reliable reception, which can be confirmed from PEP expression that larger diversity gain can be achieved when more receiving antennas are involved.

\section{Conclusion}

This article proposed a multi-cell cooperative transmission scheme based on unitary space-time modulation. Each cooperative BS sends an individual signal to a common mobile unit therefore the inter-cell CCI is eliminated and the data transmission rate increases as well. Error probability for the proposed transmission scheme is analyzed. Based on the PEP analysis, cooperative constellation optimization is presented, in which by selecting the proper constellation subset, the overall SER is reduced. Simulation results confirm the effectiveness of the proposed transmission scheme in both block-constant channels, fast fading channels and doubly-selective channels.

\section{Competing interests}

The authors declare that they have no competing interests.

\section{Acknowledgements}

This work was supported by the National Basic Research Program of China (973 Program No. 2012CB316100), the NSFC (No. 61032002 \& No. 60872014) and the 111 project (No. 111-2-14).

Received: 15 February 2012 Accepted: 12 June 2012

Published: 10 August 2012

\section{References}

1. ITU-R, Guidelines for evaluation of radio interface technologies for IMTAdvanced. Rep. M, 2135 (2008)

2. J Li, M Kavehrad, Effects of time selective multipath fading on OFDM systems for broadband mobile applications. IEEE Commun. Lett. 3(12), 332-334 (1999)

3. H Steendam, M Moeneclaey, Analysis and optimization of the performance of OFDM on frequency-selective time-selective fading channels. IEEE Trans. Commun. 47(12), 1811-1819 (1999)

4. Y Zhang, H Liu, MIMO-OFDM systems in the presence of phase noise and doubly selective fading. IEEE Trans. Veh. Technol. 56(4), 2277-2285 (2007)

5. BM Hochwald, TL Marzetta, Unitary space-time modulation for multiple-antenna communication in Rayleigh flat-fading. IEEE Trans. Inform. Theory. 46(2), 543-564 (2000)

6. BM Hochwald, W Sweldens, Differential unitary space-time modulation. IEEE Trans. Commun. 48(12), 2041-2052 (2000)

7. BL Hughes, Differential space-time modulation. IEEE Trans. Inf. Theory. 46, 2567-2578 (2000)

8. B Bhukania, P Schniter, Multiple-symbol detection of differential unitary space-time modulation in fast-fading channels with known correlation. in Conference on Information Sciences and Systems (CISS). (Princeton University, 1-6, 2002)

9. M Ramezani, M Hajiaghayi, C Tellambura, Receive antenna selection for unitary space-time modulation over semi-correlated ricean channels. IEEE Trans. Commn. 58(2), 521-530 (2010)
10. GJ Foschini, K Karakayali, Coordinating multiple antenna cellular networks to achieve enormous spectral efficiency. Proc. IEEE Commun. 153, 548-555 (2006)

11. H Zhang, H Dai, Cochannel interference mitigation and cooperative processing in downlink multicell multiuser mimo networks. EURASIP J. Wirel, Commun. Netw. 2004(2), 222-235 (2004)

12. W Hardjawana, B Vucetic, Y Li, Multi-User cooperative base station systems with joint precoding and beamforming. IEEE J. Sel. Topic Signal Process. 3(6), 1079-1093 (2009)

13. DP Liu, QT Zhang, Q Chen, Structures and performance of noncoherent receivers for unitary space-time modulation on correlated fast-fading channels. IEEE Trans. Veh. Technol. 53(4), 1116-1125 (2004)

14. BM Hochwald, TL Marzetta, TJ Richardson, W Sweldens, R Urbanke, Systematic design of unitary space-time constellations. IEEE Trans. Inf. Theory. 46(6), 1962-1973 (2000)

15. WC Jakes, Microwave Mobile Communications [M]. (Piscataway, NJ, IEEE Press, 1993)

16. Z Jia, S Handa, F Sasamori, S Oshita, Multiple-symbol differential detection for unitary space-time-frequency coding. in Conference of Circuits and Systems for Communications (ICCSC'08). (Shanghai, 139-142, 2008)

17. 3GPP TS 36.211 v910. Evolved Universal Terrestrial Radio Access (E-UTRA): Physical Channels and Modulation (Release 9)

doi:10.1186/1687-1499-2012-251

Cite this article as: Liu et al:: Multi-cell cooperative transmission based on unitary space-time modulation. EURASIP Journal on Wireless Communications and Networking 2012 2012:251.

\section{Submit your manuscript to a SpringerOpen ${ }^{\mathcal{O}}$ journal and benefit from:}

- Convenient online submission

- Rigorous peer review

- Immediate publication on acceptance

- Open access: articles freely available online

- High visibility within the field

- Retaining the copyright to your article

Submit your next manuscript at $>$ springeropen.com 